\section{La Responsabilidad Social Universitaria como estrategia de marketing en la universidad}

University Social Responsibility as a University Marketing Strategy

Carlota López-Aza Fucultad de Ciencias Económicas y Empresariales Universidad Comp
carlop14@ucm.es José-Luis Vázquez Facultad de Ciencias Económicas y Empresariales jose-luis.vazquez@unileon.es

Ana Lanero

Facultad de Ciencias Económicas y Empresariales ana.lanero@unileon.es
Clasificación JEL:

M14, M1

Palabras clave:

calidad,

satisfacción,

responsabilidad

social universitaria,

mínimos cuadrados

parciales

ABSTRACT

JEL Classification

M14, M31

Key words:

quality,

López-Aza, C., Vázquez, J.L. y Lanero, A. (2019) La Responsabilidad Social Universitaria como

aDResearch ESIC. No 19 Vol 19

Primer semestre, enero-junio 2019 . Págs. 68 a 85

Pictic-019-04
Este estudio persigue el objetivo de analizar los efectos de la percepción de los estudiantes en materia de responsabilidad social universitaria (RSU) sobre sus experiencias de calidad percibida y satisfacción, así como conocer si la RSU puede ser un antecedente que armonice la estrategia de marketing de la organización con las expectativas de sus estudiantes Tomando como referencia el modelo de los cuatro impactos universitarios, se hipotetizó un efecto directo de la valoración de los mismos sobre la percepción global de la RSU mantenida por el alumnado, que a su vez influiría positivamente sobre las dimensiones de calidad percibida y satisfacción. La investigación empírica consistió en una encuesta realizada a estudiantes de Ciencias e Ingenierías en la Universidad de León. Los datos fueron analizados a través de análisis factorial exploratorio y modelado de ecuaciones estructurales con la técnica de mínimos cuadrados parciales (partial least squares, PLS). Los resultados permitieron identificar seis factores en la percepción estudiantil de los impactos universitarios, si bien sólo tres de ellos (impacto educativo-medioambiental, impacto organizacional e impacto social) afectaron la percepción global de los estudiantes sobre RSU. Asimismo, se encontró un efecto positivo de la percepción estudiantil en materia de RSU sobre la calidad percibida y la satisfacción, las cuales también estuvieron positivamente asociadas entre sí. Los resultados del estudio apoyan la RSU como instrumento de marketing de mejora de la experiencia universitaria, y tienen importantes implicaciones para el diseño de las estrategias de marketing que permitan la mejor atracción y retención de estudiantes en entornos de educación superior.

This study aims to analyze the effects of students' perception on university social responsibility (USR) on their experiences of perceived quality of service and satisfaction, as well as to know if the USR could be an antecedent that harmonizes the university marketing strategy with the student's expectations. Using as a reference the four impacts-based model, it was hypothesized that assessment of those university impacts would have a positive effect on students' global perception of USR, which in turn would affect positively the dimensions of perceived quality and satisfaction. The empirical research consisted in a survey to a sample of students within Sciences and Techniques at the University of León. Data was analyzed with exploratory factor analysis and structural equations modelling with the partial least squares (PLS) technique. Results identified six factors in the student perception of the university impacts, although only three of these (environmental-educational impact, organizational impact and social impact) affected the student's global perception on URS. Similarly, a positive effect of students' USR global perception on their experiences of quality of service and satisfaction was found. The results of the study support the competitive advantage that USR represents as an instrument to improve the university experience, and have important implications for the design of marketing strategies to attract and retain students in higher education contexts. 


\section{Introducción}

En los últimos años, las instituciones de educación superior están trabajando en un ambiente competitivo similar al de cualquier empresa por lo que están interesadas en promover actividades de marketing al objeto de incrementar su expansión en el mercado (Geryk, 2016). Esta transformación se debe a la actual amplitud de la oferta de servicios educativos, la mayor demanda y exigencia por parte del alumnado, la reducción de fondos públicos y, en definitiva, al desarrollo de una enseñanza global que impulsa a las universidades hacia la competencia comercial (Burcea y Marinescu, 2011). Paralelamente, el Espacio Europeo de Educación Superior (EEES) ha propuesto al estudiante como eje fundamental del sistema educativo (Blázquez, Chamizo, Cano y Gutiérrez, 2013), entendiéndose además que aspectos como la calidad del servicio y la satisfacción del alumnado son imprescindibles en la consolidación del conocimiento y la innovación como impulsores de nuestro crecimiento futuro. Como muestra de ello, el objetivo de las políticas educativas, tanto europeas como nacionales, es el de mejorar los resultados y la calidad de los servicios universitarios (Comisión Europea, 2010), así como conocer la satisfacción de las expectativas y necesidades de sus stakeholders principales, los estudiantes. En ese sentido, el verdadero éxito de la estrategia de marketing universitaria debe ser su orientación desde el exterior al interior, partiendo de las necesidades de sus estudiantes para posteriormente preparar una oferta de servicios de educación superior adecuados (Geryk, 2016).

Con todo, la calidad pasa a ser un factor esencial para el éxito de las universidades (Landrum, Prybutok y Zhang, 2007) por sus efectos sobre la retención de los estudiantes (Russell, 2005) y la labor de recomendación generada por alumnos satisfechos que ven su universidad como insti- tución de confianza. En este sentido, la relación entre calidad y satisfacción ha sido ampliamente estudiada en la literatura previa, que apoya la existencia de una la relación positiva y directa entre ambos constructos (Arambewela y Hall, 2008; Ben, 2007; Boshoff y Gray, 2004). De esta manera, esa relación podría cumplirse también en las universidades de hoy en día, las cuáles son conscientes de la necesidad de mantener a sus estudiantes satisfechos con su experiencia universitaria como forma de conseguir su lealtad (Sánchez-Hernández y Mainardes, 2016).

Asimismo, la preocupación por la calidad en el ámbito universitario se ha extendido desde el análisis de los contenidos pedagógicos y el proceso de enseñanza en general a otros niveles (Alashloo, Castka y Sharp, 2005), fundamentalmente al estudio de los antecedentes (Abdullah, 2005; Shekarchizadeh, Rasli y Hon-Tat, 2011). A tal respecto, mientras algunos autores han propuesto la información y comunicación, las referencias sociales y las creencias de los usuarios como determinantes clave (Gournaris, Stathakopoulos y thanassopoulos, 2003), otros investigadores se basan en marcos teóricos bien establecidos, como el modelo Servqual (Bigné, Molinar y Sánchez, 2003; Kilbourne, Duffy, Duffy y Giarchi, 2004). En este nuevo contexto, las universidades deberían adoptar una nueva estrategia responsable orientada a sus estudiantes para diferenciar su oferta de la de sus competidores. La satisfacción estudiantil está en profundos cambios (SánchezHernández y Mainardes, 2016) lo que provoca la necesidad de buscar nuevos antecedentes que contribuyan a cambiar la cultura universitaria a na cultura de responsabilidad

Ampliando los estudios previos, la contribución de este estudio es proponer el concepto de responsabilidad social universitaria (RSU) como un impulsor de la calidad percibida y satisfacción de los estudiantes, a partir de la literatura previa que apoya tal relación en contextos empresariales y comerciales (Huang, Yen, Liu, y Huang 2014; Loureiro y González, 2012; Mandhachitara y Poolthong, 2009;) y también en contextos educativos (Perić y Delić, 2016; Plungpongpan, Tiangsoongnern y Speece, 2016). En definitiva, investigadores como Maignan y Ferrel (2001) ya sugirieron utilizar la responsabilidad social como una herramienta de marketing que permitiera crear valor a una organización, de ahí que su adopción por parte de la universidad con un enfoque estratégico conduzca actualmente a la promoción de una estrategia de marketing que pueda asegurar la supervivencia de la universidad en el mercado (Geryk, 2016).

Para ello, se parte del modelo de RSU propuesto por Vallaeys $(2006,2014)$, que identifica cuatro tipos de impactos generados por el desarrollo de la labor universitaria: el impacto educativo, que fomenta la formación profesional y ciudadana responsable; el impacto cognitivo, que promueve la gestión social del conocimiento; el impacto organizacional, que impulsa los campus responsables; y el impacto social, que aviva la participación de la comunidad universitaria. A pesar de que se trata de un modelo de referencia en la literatura previa, la mayor parte de trabajos se han centrado en el impacto educativo de las universidades (Balotsky y Steingard, 2006; Caldwell 2009; Christensen, Peirce, Hartman, Hoffman y Carrier, 2007; Hartman y Werhane, 2009; Larrán y Andrades, 2014; McDonald, 2004; Moon y Orlitzky, 2011), mientras que las tres dimensione restantes apenas han sido consideradas (López Aza, 2016; Vázquez et al., 2015).

Los resultados previos solo hacen referencia al aumento progresivo de las asignaturas responsables en los diferentes grados gracias al proceso de Bolonia y al Espacio Europeo de Educación Supe- rior (Larrán y Andrades, 2014) pero al ser estudios descriptivos centrados en el impacto educativo no están presentes los otros tres impactos que conforman la RSU ni las percepciones de los estudiantes, los cuáles son el eje fundamental de la misión universitaria. Este estudio contribuye a ambas líneas de investigación mediante la propuesta de un modelo de impactos universitarios para explicar la percepción del alumnado técnico y científico con respecto a la RSU y su efecto en la configuración de su experiencia universitaria en términos de calidad percibida y satisfacción. En definitiva, la principa contribución de esta investigación a la literatura existente es exponer la RSU como una fuente de ventaja competitivas para la estrategia de marketing universitaria utilizando como principal stakeholder a los estudiantes teniendo en cuenta que la responsabilidad social es parte de la habilidades conocimientos y aptitudes que cualquier persona debe adquirir para un futuro sostenible.

Para obtener información sobre la relevancia de la RSU en la calidad y satisfacción de los estudiantes, como principal parte interesada de la universidad, el artículo se organiza de la siguiente manera. En primer lugar, se analizan las dimensiones de calidad y satisfacción, así como las interrelaciones entre ambos constructos. La segunda sección del trabajo examina el modelo de RSU de los cuatro impactos (Vallaeys, 2006, 2014) y su propuesta como antecedente para explicar la experiencia universitaria (calidad y satisfacción). A continuación, se describe el diseño y resultados de un estudi empírico realizado en una universidad española. Finalmente, se aporta una sección de conclusiones y líneas de investigación futura.

\section{Calidad percibida y satisfacción}

\section{universitaria}

Como instituciones que proporcionan el nive educativo más elevado, las universidades se en- 
frentan hoy día a una gran presión para conseguir el éxito (Prasad y Jha, 2013), debiendo preocuparse no sólo de la formación que reciben sus estudiantes, sino también de atraer nuevos alumnos y retener a los que ya lo son garantizando una buena experiencia global. En este sentido, y dado que la educación superior cumple con las características clásicas de los servicios (Zeithaml y Bitner, 2002), los conceptos de calidad y satisfacción del cliente son directamente aplicables a las universidades en su acercamiento a las necesidades de su público objetivo (Arambewela y Hall, 2008; Ben, 2007; Boshoff y Gray, 2004). Desde este punto de vista, entendemos el concepto de calidad universitaria como «la diferencia entre lo que un estudiante espera recibir y sus percepciones de la entrega real» (O'Neill y Palmer, 2004, p. 42) y el concepto de satisfacción como el «resumen evaluativo de la experiencia educativa directa basada en la discrepancia entre las expectativas previas y el rendimiento percibido después de pasar por el ciclo educativo» (Munteanu, Ceobanu, Bobâlca y Anton, 2010, p. 125).

Las definiciones anteriores reflejan que la percepción de la calidad se produce al mismo tiempo que el suministro y el consumo (Mele, 2003), momento crucial para la satisfacción. Por lo tanto, existe una estrecha conexión entre ambos conceptos. De ahí que la mayoría de los estudios previos en el contexto educativo muestren a la calidad percibida como antecedente de la satisfacción (Qureshi, Shaukat y Hijazi2010; Tan y Kek, 2004). El mismo tipo de relación es avalada por la literatura sobre servicios en general (Hanaysha, Abdullah y Warokka,2011; Hasan, Ilias, Rahman y Razak,2008) y los índices de satisfacción más conocidos, tales como el modelo americano ACSI ${ }^{1}$ (Fornell, Johnson, Anderson,
Cha y Bryant, 1996), el modelo europeo ECSI ${ }^{2}$ (Eklöf, 2000) y los dos modelos noruegos NCSB y NCSB2 ${ }^{3}$ (Andreassen y Leidestad, 1998; Johnson, Gustafsson, Andreassen, Lervik y Cha, 2001). Por tanto, partimos en nuestro modelo de la siguiente hipótesis:

- H1: la calidad percibida influye positiva y directamente sobre la satisfacción del estudiante (ver Figura 1).

\section{La RSU como antecedente de} calidad percibida y satisfacción

\section{En la literatura sobre servicios en general, diversos} autores asocian la disponibilidad de información en materia de responsabilidad social empresarial (RSE) con la mayor calidad percibida y satisfacción del consumidor (Huang et al., 2014; Loureiro y González, 2012; Mandhachitara y Poolthong, 2009). En cuanto a la literatura sobre servicios educativos, investigaciones previas concluyen que la educación de calidad universitaria es un requisito previo para el desarrollo de la RSU en la conciencia de los jóvenes estudiantes

(Perić y Delić, 2016) y una medida de control de la calidad univ ersitaria (Plungpongpan, Tiangsoongnern y Speece, 2016). Es más, los cambios en el mercado de la educación superior han provocado que la competencia basada en la imagen de la universidad esté ganando importancia a la hora de que un estudiante elija su universidad (Geryk, 2016). Por tanto, la orientación responsable de una universidad puede y debe convertirse en un elemento clave en las percepciones de calidad y satisfacción por parte del alumnado ya que está considerada una estrategia de gerencia ética e inteligente de los impactos de la organización en su entorno humano, social y natural (Garde-Sánchez, Rodríguez-Bolívar y López-Hernández, 2013), así como una fuente de superioridad posicional y de ventaja competitiva (Brown y Mazzarol, 2009).

Partiendo de tales evidencias, proponemo en este estudio a la RSU como antecedente de la calidad y satisfacción universitarias. Para ello, tomamos como referencia la definición de RSU propuesta por Vallaeys (2006, p. 11): «Politica de gestión de calidad ética de la universidad que busca alinear sus cuatro procesos (gestión, docencia, investigación, extensión) con la misión universitaria, sus valores y compromiso social, mediante el logro de la congruencia institucional, la transparencia y la participación dialógica de toda la comunidad universitaria».

Desde esta perspectiva, se plantea que una percepción positiva de la RSU por parte del alumnado mejorará la experiencia universitaria, logrando instituciones universitarias de mayor calidad y con unos estudiantes más satisfechos (Vázquez et al. 2015). Asimismo, el mercado competitivo de la educación superior enfoca a las universidades a satisfacer a sus estudiantes y la RSU es un concepto que causa motivación, satisfacción y retención (Sánchez-Hernández y Mainardes, 2016). Sin embargo, el predominio de los estudiantes como stakeholders universitarios no está en consonancia con la cantidad de artículos académicos que analizan la relación entre la experiencia universitaria y la RSU, los cuáles son paradójicamente escasos. Por consiguiente, se proponen las siguientes hipótesis de investigación:

- H2: La percepción global de la RSU influye positiva y directamente sobre la calidad percibida (ver Figura 1)

- H3: La percepción global de la RSU influye positivamente sobre la satisfacción del estudiante, de forma directa (ver Figura 1)
A la hora de explicar la formación de la percepción global de la RSU por parte del alumnado, recurrimos al modelo de impactos universitarios de Vallaeys $(2006,2014)$, vinculado al modo en el que las organizaciones gestionan los efectos que causan en su entorno. Desde esteenfoque, se asume que las universidades deben generarcuatro tipos de impactos positivos: i) impacto educativo, relativo alaformación de profesionales y ciudadanos responsables; ii) impacto cognitivo, referente al tipo de conocimientos generados por la investigación universitaria, su pertinencia social y sus destinatarios; iii) impacto organizacional, relativo al ámbito interno del personal universitario y los estudiantes, y a la consideración de aspectos medioambientales en la gestión de recursos; y iv) impacto social en relación a la promoción del desarrollo de la sociedad mediante la colaboración para resolver problemáticas fundamentales

La mayoría de las investigaciones previas en esta materia se han desarrollado desde la perspectiva del profesorado y los gestores universitarios, dejando a un lado a los estudiantes (Joseph, Yakhou y Stone, 2005), y han estado centrada exclusivamente en el impacto educativo, tratándolo además desde un punto de vista eminentemente descriptivo y pedagógico (Balotsky y Steingard, 2006; Caldwell, 2009; Christensen et al., 2007; Hartman y Werhane, 2009; Larrán y Andrades, 2014; McDonald, 2004; Moon y Orlitzky, 2011). Frente a ello, existe un escaso conocimiento tanto teórico como empírico sobre la percepción mantenida por alumnos con respecto a la actividad de las universidades en los tres impactos restantes: cognitivo, organizacional y social. Atendiendo a tal estado de la investigación, planteamos aquí la validación empírica de los cuatro factores identificados por el modelo de impactos (Vallaeys, 2006) en la explicación de la 


\section{percepción de los estudiantes con respecto a la Metodología}

RSU. Particularmente, proponemos las siguientes hipótesis:

- H4a: La percepción del impacto educativo influye positiva y directamente sobre la percepción global de la RSU (ver Figura 1)

- H4b: La percepción del impacto cognitivo influye positiva y directamente sobre la percepción global de la RSU (ver Figura 1)

- H4c: La percepción del impacto organizacional influye positiva y directamente sobre la percepción global de la RSU (ver Figura 1)

- H4d: La percepción del impacto social influye positiva y directamente sobre la percepción global de la RSU (ver Figura 1)

\section{Figura 1 • Hipótesis del modelo planteado}

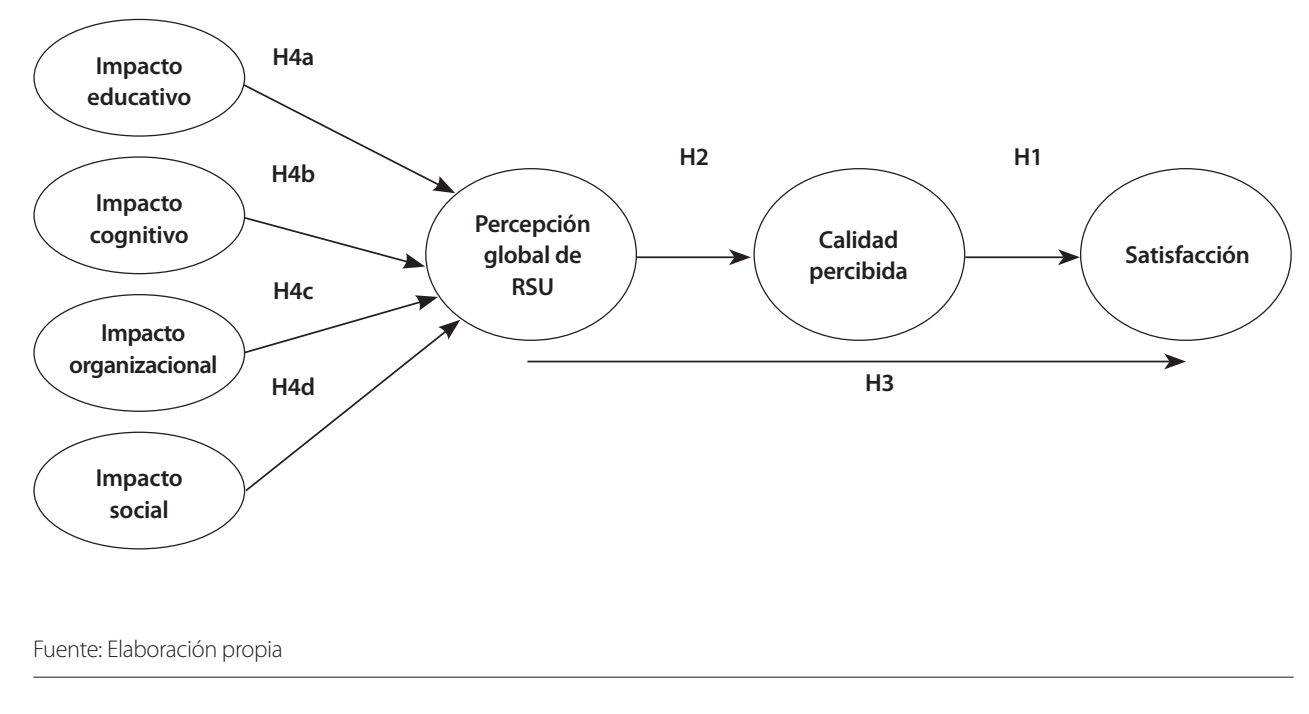

mayor amplitud de la experiencia universitaria. Por lo que respecta a las características sociodemográficas, la muestra total estuvo compuesta po 85 hombres ( $42.5 \%$ ) y 115 mujeres (57.5\%), con edades comprendidas entre los 20 y los 32 años de edad ( $M=22,33 ; D T=2,273)$.

El cuestionario administrado a la muestra seleccionada fue construido para los propósitos de esta investigación a partir de una profunda revisión de la literatura especializada, y fue refinado en base a la opinión de tres expertos en el área (López- Aza, 2016). La calidad percibida fue medida con 5 ítems basados en la literatura previa sobre calidad del servicio en la educación superior (Abdullah, 2005; Loureiro y González, 2012). Para medir la satisfacción, se emplearon 6 ítems relativos a actuaciones universitarias en diferentes áreas (Andreassen y Lindestad, 1998 Eklöf, 2000, Fornell et al., 1996). Para los dos constructos, los encuestados debían indicar su grado de acuerdo con cada enunciado en escala Likert de 1 (totalmente en desacuerdo) a 5 (totalmente de acuerdo).

La percepción global de los estudiantes sobre la RSU fue medida con tres ítems relativos a la valoración de la institución al triple nivel social, económico y medioambiental. De nuevo, se empleó una escala de respuesta tipo Likert de 1 (totalmente en desacuerdo) a 5 (totalmente de acuerdo). La percepción de los estudiantes con respecto a los impactos universitarios se estimó a partir de un banco inicial de 46 ítems basados en la revisión de trabajos previos y de otros instrumentos similare (Christensen et al., 2007; McDonald, 2004; Moon y Orlitzky, 2011; Vallaeys, 2006, 2014). Del total, 12 ítems se refirieron al impacto educativo, definiendo actuaciones responsables en materia de educación. El impacto cognitivo quedó definido por 10 ítems sobre prácticas de responsabilidad universitaria en materia de investigación. Para medir el impacto organizacional se emplearon 12 enunciados sobre actuaciones responsables en materia de gestión interna universitaria. Final mente, 12 ítems se refirieron al impacto social, definiendo actuaciones en materia de proyección social y extensión universitaria. Los participantes debían indicar su opinión sobre la importancia otorgada por la universidad a cada práctica, siguiendo una escala Likert de 1 (importancia mínima) a 5 (importancia máxima).

El cuestionario fue administrado colectivamente a los estudiantes en clases universitarias programadas, contando con el permiso del profesor responsable en cada caso y en presencia de un investigador formado en la investigación. En todo caso, los participantes fueron informados sobre el anonimato y carácter voluntario de la encuesta. E tiempo medio de respuesta al cuestionario fue de 15 minutos. Se obtuvieron 12 cuestionarios con respuestas incompletas que fueron eliminados de análisis posteriores.

El tratamiento estadístico de los datos estuvo dirigido a analizar la fiabilidad y validez de las medidas incluidas en el cuestionario y el cumplimiento de las hipótesis de investigación. La información fue procesada mediante el análisis factorial para la fase exploratoria y las ecuaciones estructurales para la fase confirmatoria. En estos análisis se utilizaronlos programas informáticos SPSS 22 y SmarPLS 2.0 (Ringle, Wende y Will, 2005), respectivamente.

En primer lugar, se realizó un análisis factorial exploratorio para evaluar la validez de constructo de las escalas incluidas en el cuestionario ${ }^{4}$. Los resultados avalaron la unidimensionalidad de las variables de calidad, satisfacción, y percepción global de la RSU.En cuanto a la percepción de impactos universitarios, el análisis de compo-

4 Los resultados están disponibles bajo petición a los autores 
nentes principales reveló una solución de seis factores que explicaron el 50,32\% de la varianza total. Los impactos cognitivo, organizacional y social se mantuvieron en esta solución, quedando definidos por cinco, cuatro y seis ítems, respectivamente. Por su parte, el impacto educativo quedó mejor representado por tres dimensiones relativas a educación en valores sociales (tres ítems), educación en valores medioambientales cuatro ítems) y educación para la mejora de la inserción laboral y el desarrollo regional (3 ítems). Tales dimensiones fueron denominadas, respectivamente, impacto educativo-social, impacto educativo-medioambiental, e impacto educativo-económico. Los 21 ítems restantes denotaron un mal funcionamiento en el análisis factorial y fueron eliminados del análisis.

A continuación, se utilizó la técnica de mínimos cuadrados parciales (partial least squares, PLS) para analizar el funcionamiento del modelo de investigación propuesto. Frente a los métodos de modelado estructural basados en el análisis de la covarianza, la técnica PLS tiene como objetivo maximizar la varianza explicada por las

variables observadas y latentes, presentando como ventajala mejor adaptación a investigaciones exploratorias y a constructos formativos (Chin, 1998a).Desde este encuadre, el procedimiento PLS permite estimar el modelo de investigación en dos etapas (Hulland, 1999).La primera de ellas implica evaluar la fortaleza del modelo de medida en términos de fiabilidad individual de los ítems, consistencia interna de las escalasy validez convergente y discriminante. La segunda etapa se centra en el modelo estructural, informando sobre l cumplimiento de las hipótesis de investigación y el ajuste global del modelo empírico.

\section{Resultados}

La Tabla I muestra las propiedades psicométricas de los 10 constructos finalmente incluidos en el modelo de medida. La fiabilidad individual de los ítems fue valorada examinando la significación de las cargas factoriales estandarizas $(\lambda)$. Todas excedieron el nivel mínimo de .50 requerido para la aceptación (Barclay, Higgins y Thompson, 1995; Chin, 1998a), calculado mediante un procedimiento bootstrap con 500 submuestras de 200 casos.

\section{Tabla I · Fiabilidad y validez del modelo de medida}

\begin{tabular}{|c|c|c|c|c|}
\hline Constructos & $\lambda$ & a & $\rho c$ & AVE \\
\hline Calidad percibida & & .81 & .87 & .57 \\
\hline Los programas académicos de mi universidad tienen una alta calidad & $.81^{* * * *}$ & & & \\
\hline El profesorado de mi universidad desempeña un trabajo de calidad & $.78^{* * *}$ & & & \\
\hline El personal administrativo de mi universidad desempeña un trabajo de calidad & $.69 * * *$ & & & \\
\hline Mi universidad cuenta con recursos e instalaciones de alta calidad & $.62^{* * *}$ & & & \\
\hline En comparación con otras similares, mi universidad ofrece unos servicios de calidad & $.78^{* * *}$ & & & \\
\hline Satisfacción & & 9 & .92 & 67 \\
\hline La experiencia que estoy teniendo en mi universidad cubre enteramente mis expectativas & $.83^{* * * *}$ & & & \\
\hline Mi decisión de escoger esta universidad fue acertada & $.80^{* * *}$ & & & \\
\hline Estoy satisfecho con la formación recibida en la universidad & $.87^{* * * *}$ & & & \\
\hline Estoy satisfecho con mi experiencia global en la universidad & $.81^{* * *}$ & & & \\
\hline Recomendaré esta universidad a otras personas & $.86^{* * *}$ & & & \\
\hline Me siento orgulloso de pertenecer a esta universidad & $.82^{* * *}$ & & & \\
\hline
\end{tabular}

Percepción global de la RSU

Mi universidad tiene un elevado potencial para contribuir a l respeto y protección del

medioambiente Mi universidad tiene un elevado potencial para contribuir al crecimiento y desarrollo económico Impacto educativo-social

Consideración de la opinión y participación de los alumnos

Formación en valores humanos y sociales y fomento de la solidaridad ciudadan

Respeto a la diversidad e iqualdad de oportunidades entre el alumnado

\section{Impacto educativo-medioambiental}

Concienciación de la comunidad universitaria sobre problemas medioambientales

Formación técnico-profesional para la solución de problemas medioambientales

Enseñanza de hábitos ecológicos y valores de sostenibilidad

Cuidado y conservación de zonas ecológicas de la universidad

\section{mpacto educativo-económico}

Fomento del espiritu emprendedor de los estudiantes

Colaboración con empleadores para mejorar la formación en inserción laboral

Transferencia de conocimientos hacia la empresa

\section{Impacto cognitivo}

Incorporación de principios sostenibles en la realización de investigaciones cientificas

Conducción de investigación en materia de sostenibilidad

Aplicación del conocimiento científico al desarrollo de nuevos productos, tecnologías y procesos respetuosos con el medio ambiente

Integración de principios de respeto a los derechos individuales y sociales en las investigaciones Investigación de problemas sociales y generación de conocimientos para el desarrollo socia Impacto organizacional

Conciliación de la vida laboral y familiar del profesorado y personal administrativo

Elección transparente y democrática de las autoridades y gestión participativa

Respeto a la diversidad y promoción de igualdad de oportunidades entre el personal

Asignación racional y eficiente de los recursos

Impacto social

Campañas de sensibilización, educación y protección ambiental

Colaboración con empresas, AA.PP y/u ONGs en iniciativas sostenibles

Campañas de sensibilización y educación sobre responsabilidad social

Organización de programas de voluntariado para estudiantes, profesores y personal administrativo

Colaboración con empresas, AA.PP y/u ONGs en proyectos sociales de ayuda a colectivos

Patrocinio y organización de actuaciones comprometidas con el desarrollo socioeconómico local

Los resultados están disponibles bajo petición a los autores.

$\lambda$ (cargas factoriales): a (alpha de Cronbach); pc (fiabilidad compuesta): AVE (varianza media extraida):*** $<001$ (t de Student con 499 grados de libertad). 
Por otra parte, la fiabilidad de los construc- Para comprobar tal condición, la Tabla II expotos fue examinada mediante los índices alpha de ne la matriz de correlaciones entre las variables Cronbach (a) y fiabilidad compuesta $(\rho)$, que latentes, reemplazando la diagonal por la raíz indican la consistencia interna de las escalas. Am- cuadrada de los valores AVE, los cuales fueron bos índices son interpretados de manera similar, superiores al resto de índices $r$ (Fornell y Larcker, considerándose apropiadas los valores por enci- 1981)

ma de 70 (Barclay et al., 1995). Por otra parte, la Una vez que se comprobó la fiabilidad y valivalidez convergente fue estimada por la varianza dez del modelo de medida, se utilizó el programa extraída media (average variance extracted, AVE), PLS para poner a prueba las relaciones hipotetiestando por encima del nivel de referencia de .50 zadas entre las variables latentes exógenas y enpara todos los constructos del modelo (Fornell dógenas. Se aplicó un procedimiento de bootstrap y Larcker, 1981).Del mismo modo, para poner con 500 submuestras para obtener los valores del a prueba la validez discriminante, se verificó estadístico $t$ (Chin, 1998a) de cada ruta estimada que cada constructo compartiera una varianza en el modelo. La Tabla III muestra los efectos más grande con sus indicadores que con otras directos obtenidos y sus correspondientes niveles dimensiones del modelo (Barclay et al., 1995). de significación.

\section{Tabla II · Matriz de correlaciones y raíz cuadrada de AVE (diagonal)}

\begin{tabular}{|c|c|c|c|c|c|c|c|c|c|}
\hline & 1 & 2 & 3 & 4 & 5 & 6 & 7 & 8 & 9 \\
\hline 1. Calidad percibida & .75 & & & & & & & & \\
\hline 2. Satisfacción & $.79 * *$ & .82 & & & & & & & \\
\hline 3. Percepción global de RSU & $.57 * *$ & 64.** & .83 & & & & & & \\
\hline 4. Impacto educativo-social & $.15^{* *}$ & $.31 * *$ & $.44^{* *}$ & .77 & & & & & \\
\hline 5. Impacto educativo-medioambiental & $.14^{* *}$ & $.10 * *$ & $.36^{* *}$ & $.51 * *$ & .73 & & & & \\
\hline 6. Impacto educativo-económico & $.06^{* *}$ & $.13^{* *}$ & $.42^{* *}$ & .61** & $.49^{* *}$ & .72 & & & \\
\hline 7. Impacto cognitivo & $.31 * *$ & $.30^{* *}$ & $.42^{* *}$ & $.53^{* *}$ & $.54^{* *}$ & $.53 * *$ & .72 & & \\
\hline 8. Impacto organizacional & $.25^{* *}$ & $.32^{* *}$ & $.33^{* *}$ & $.42^{* *}$ & $.44^{* *}$ & $.42^{* *}$ & $.50^{* *}$ & .72 & \\
\hline 9. Impacto social & $.14^{* *}$ & $.15 * *$ & $.38^{* *}$ & $.47^{* *}$ & $.66^{* *}$ & $.52^{* *}$ & $.34^{* *}$ & $.40 *$ & .75 \\
\hline$p<.05 ; * * 0<.005 ; * * * p<.001$. & & & & & & & & & \\
\hline
\end{tabular}

De acuerdo al planteamiento de la H1, la calidad percibida tuvo un efecto positivo directo sobre la satisfacción del estudiante $(\beta=.63 ; p<.001)$ Por lo que respecta a la $\mathrm{H} 2$, se observó también un efecto positivo directo de la percepción global de la RSU sobre la calidad $(\beta=.57 ; p<.001)$. Por tanto, se han probado dos vías de impacto de la
RSU hacia la satisfacción, una mediada por la calidad del servicio y otra no.

Igualmente, en apoyo a la H3, la percepción global de la RSU tuvo un efecto positivo directo sobre la satisfacción $(\beta=.29 ; \beta<.01)$.

La H4a referida a los efectos del impacto educativo únicamente obtuvo apoyo para el factor

\section{Tabla III · Ajuste del modelo estructural}

\begin{tabular}{|l|c|}
\multicolumn{1}{|c|}{ Hipótesis } & $\begin{array}{c}\text { Efectos directos } \\
(\beta)\end{array}$ \\
\hline H1: Calidad percibida $\rightarrow$ Satisfacción & $.63^{* * *}$ \\
\hline H2: Percepción global de RSU $\rightarrow$ Calidad percibida & $.57^{* * *}$ \\
\hline H3: Percepción global de RSU $\rightarrow$ Satisfacción & $.29^{* *}$ \\
\hline $\begin{array}{l}\text { H4a: Impacto educativo-social } \rightarrow \text { Percepción global de RSU } \\
\quad \text { Impacto educativo-medioambiental } \rightarrow \text { Percepción global de RSU }\end{array}$ & $.18^{*}$ \\
\hline $\begin{array}{l}\text { Impacto educativo-económico } \rightarrow \text { Percepción global de RSU } \\
\text { H4b: Impacto cognitivo } \rightarrow \text { Percepción global de RSU }\end{array}$ & $.19^{*}$ \\
\hline H4c: Impacto organizacional $\rightarrow$ Percepción global de RSU & $.05(\mathrm{~ns})$ \\
\hline H4d: Impacto social $\rightarrow$ Percepción global de RSU & $.16^{*}$ \\
\hline
\end{tabular}

${ }^{*} p<.05 ; * * *<<005 ; * * * p<.001$; ns: no significativo (t de Student con 400 grados de libertad).

relativo a la educación en valores sociales, que se asoció positiva y directamente con la percepción global de la RSU $(\beta=.18 ; p<.05)$. No obstante, el modelo no proporcionó apoyo empírico a los efectos de los impactos educativo-social y educativo-económico sobre las dimensiones estudiadas. Tales resultados proporcionan un apoyo limitado a la hipótesis. En la misma línea, la H4c no obtuvo apoyo empírico, no encontrándose evidencia de efecto significativo del impacto organizacional sobre la percepción global de RSU.

Por su parte, los impactos cognitivos (H4b) y social $(\mathrm{H} 4 \mathrm{~d})$ aparecieron asociados a efectos significativos directos sobre la percepción global de la RSU $(\beta=.19 ; p<.05$ y $\beta=.16 ; p<.05$, respectivamente) e indirectos sobre la calidad ( $\beta$ $=.13 ; p<.001$ y $\beta=.16 ; p<.001$ ) y la satisfacción $(\beta=.03 ; p<.001$ y $\beta=.04 ; p<.001)$.

En el contexto de tales resultados, la capacidad predictiva del modelo fue valorada mediante el estadístico R2(Figura I), que debe superar el valor 10 (Falk y Miller, 1992). Con todo, el modelo global permitió explicar el 32\% de la varianza en la calidad percibida, el 68\% en la satisfacción y el
28\% en la percepción global de la RSU. Del mismo modo, el ajuste global del modelo en términos de relevancia predictiva se estimó a través de un procedimiento jackknife basado en la prueba de Stone-Geisser (Geisser, 1974; Stone, 1974). Los valores 22 recomendados han de ser positivos y superiores a .10 (Chin 1998b). Ambos requisitos fueron satisfechos para la calidad percibida ( $2=.10)$, la satisfacción $(Q 2=.10)$ y la percepción global de la RSU (Q2=.12).

\section{Conclusiones}

En la actualidad, las universidades están trabajando en entornos competitivos muy dinámicos, por lo que necesitan encontrar ventajas competitivas para fortalecer sus oportunidades de liderazgo en el mercado. En esta situación, las universidades deben verse a sí mismas no como un proveedor de de servicios educativos de alta calidad, sino como una institución que satisface todas las necesidades de su principal stakeholder, el estudiante (Geryk, 2016). De ahí que la calidad del servicio sea un factor esencial para el éxito, especialmente como medio para conseguir estudiantes satisfe- 
chos que recomienden su universidad a otros (Landrum et al., 2007). Desde este encuadre, los resultados de este estudio van en la línea dela literatura previa (Hanaysha et al., 2011; Hasan et al., 2008) que evidencia el efecto positivo de la calidad percibida sobre la satisfacción del alumno, reforzando la idea de que ambos son constructos inseparables que previsiblemente se asociarán a niveles elevados de retención estudiantilllevando, en definitiva, a las universidades a ser vistas como instituciones de confianza (Qureshi et al., 2010; Tan y Kek, 2004).

Partiendo de lo anterior, la presente investigación ha tratado de ahondar en los desencadenantes de la calidad percibida y la satisfacción estudiantil, proponiendo la RSU como elemento diferenciador dentro del mercado educativo y analizando su impacto sobre las dimensiones de experiencia universitaria. En este sentido, los resultados apoyan la idea de que una percepción positiva global de los estudiantes con respecto a la contribución socioeconómica y medioambiental de la institución en su entorno se asocia a mayores índices de calidad subjetiva, lo que a su vez refuerza la experiencia de satisfacción. Por tanto, queda claro que una estrategia de marketing basada en la mejora de la imagen de la universidad a través de herramientas de comunicación que fomente la responsabilidad social parece ser la más relevante en términos de ventaja competitiva que ayude a los estudiantes a decidir entre instituciones, llegando a considerar la implicación institucional en prácticas responsables como criterio de calidad y aval de experiencia satisfactoria. Asimismo, se han encontrado apoyos para una relación directa entre la RSU y la satisfacción sin la mediación de la calidad del servicio, lo cual está en consonancia con estudios previos (SánchezHernández y Mainardes, 2016) donde el vínculo entre la RSU y la satisfacción estudiantil puede ser un factor importante que contribuya a una cultura universitaria más responsables y por tanto confirma la hipótesis de la RSU como antecedente de la experiencia universitaria.

Esta evidencia debe ser considerada como fuente de información útil para las estrategias de marketing universitario, conduciendo a medio plazo a la mejora del prestigio y notoriedad institucional, reduciendo el abandono estudiantil, fomentando la atracción de alumnado nuevo. Esto significa que la tarea de marketing debe ser crear una imagen de marca responsable que pueda contribuir al aumento del valor de toda la organización

Avanzando tales planteamientos, este trabajo proporciona ciertas pistas sobre la configuración de la percepción global de RSU en los estudiantes. A tal respecto, los resultados obtenidos proporcionan un apoyo empírico relativo al modelo de los cuatro impactos de Vallaeys $(2006,2014)$. Si bien la diferenciación entre los impactos cognitivo, organizacional y social propuestos inicialmente por el autor sí resultó útil para dar cuenta de la percepción que el alumnado mantiene con respecto a la RSU al igual que investigaciones previas (López-Aza, 2016; Vázquez, López Aza y Lanero, 2015), el análisis factorial exploratorio realizado resultó más afín a una solución de seis impactos, apuntando a la conveniencia de segmentar el impacto educativo en las tres facetas social, medioambiental y económica. Este resultado está, no obstante, en plena consonancia con el modelo de la Triple Bottom Line de Elkington (1997), que identifica las mismas dimensiones en su propuesta teórica del concepto de responsabilidad social en contextos empresariales. Desde este punto de vista, podemos concluir que la triple cuenta de resultados se puede generalizar al modelo de RSU, con el fin de obtener una mejor comprensión de las experiencias de los estudiantes respecto a la educación responsable en línea con los resultados de (López Aza, 2016; Vázquez et al., 2015)

Partiendo de la anterior identificación de impactos universitarios, los resultados de la investigación también ponen de manifiesto que no toda las prácticas universitarias responsables son percibidas como tales o tienen la misma relevancia para los alumnos. En concreto, se obtuvo evidencias de apoyo significativo para los impactos educativosocial, cognitivo, y social, sobre la percepción global de RSU. En cuanto al primero de ellos, los estudiantes tienen cada vez más asignaturas responsables en sus currículos académicos o temas integrados dentro de asignaturas relacionadas con la responsabilidad social, debido a que es uno de los objetivos del Espacio Europeo de Educación Superior (Larrán y Andrades, 2014). Por tanto es coherente que el impacto educativo-social sea significativo, pero todavía no reconocido en el resto de impactos educativos, debiéndose a que la integración de la responsabilidad social en e currículo es un fenómeno relativamente nuevo, multifacético y en continuo cambio (Setó-Pamiés et al., 2011). En cuanto al apoyo al impacto cog nitivo, los estudiantes de ingenierías y ciencias están muy involucrados en la investigación aplicada (descubrimientos científicos a problemas, y/o productos comerciales) por tanto es un área en la que son conscientes de la importancia de investigaciones responsables y va en consonancia con la importancia atribuida a la vinculación enseñanza-investigación responsable (SánchezHernández y Mainardes, 2016). La explicación del apoyo al impacto social está en consonancia con los resultados de (Vázquez et al., 2015) quienes argumentaban que las facultades de carrera relacionadas con la empresa están más estrechamente relacionadas con instituciones que proponen campañas de comunicación, patrocinio, etc. en el ámbito de la proyección social lo que se puede extrapolar a las Facultades de Ingeniería y Ciencias cuya relación con la empresa privada es muy importante.

Por otro lado, cabe destacar que no se obtuvo evidencia de apoyo empírico a la relación directa entre la valoración que los estudiantes otorgan a los impactos organizacionales, educativo-económico y educativo- medioambiental, y la percepción global de la RSU. La explicación a este patrón de resultados puede venir en el caso de los impactos relacionados con la formación, que el colectivo estudiantil de ingeniería y ciencias no ha tenido una formación en responsabilidad social adecuada ni en términos medioambientales ni económicos, no reconociendo como responsables muchas de las prácticas implementadas por la universidad en su actividad cotidiana. Más bien, tales prácticas podrían ser entendidas como obligaciones básicas inherentes a la misión universitaria que, por tanto, no contribuirían en gran medida al incremento de su percepción de calidad de servicio y su posterior satisfacción. Todo ello denota que tal vez el concepto de RSU no esté siendo interiorizado adecuadamente por los estudiantes, o que la propia institución no comunica adecuadamente sus acciones responsables, lo que dificulta la identificación de las mismas por parte del colectivo estudiantil.

Retomando la explicación apuntada en el párrafo anterior, las prácticas englobadas en estos tres factores podrían resultar menos llamativas para los estudiantes por estar más alejadas de sus expectativas sobre la misión esperable de la universidad en su campo de estudios. Este hecho podría estar llevando a los estudiantes a mantener una visión limitada del concepto de RSU que debería ser ampliada mediante una adecuada comunicación de las prácticas de responsabilidad implementadas en los diferentes ámbitos de im- 
pacto institucional a través de un diseño de una estrategia de marketing adecuada a las necesidades y expectativas de este público objetivo.

En cualquier caso, lo que sí puede concluirse a la vista de los resultados del estudio es que el concepto de RSU mantenido por los alumnos y la valoración que hacen de la implicación institucional en prácticas de responsabilidad afecta a la calidad percibida y la satisfacción. A la vista de este planteamiento, parece que la RSU puede considerarse un elemento con elevado potencial para incrementar la competitividad de las universidades en el mercado de la educación superior. Por tanto, se refuerza la importancia de trabajar hacia una mejor integración transversal de prácticas responsables en todas las labores universitarias, que además sean efectivamente comunicadas a los estudiantes a través de una estrategia de marketing para que puedan identificarlas fácilmente en todos los impactos universitarios, y no sólo en los que resultan más salientes en su área de estudios. Es decir, los esfuerzos en marketing universitario no deben estar dirigidos solo a la promoción de servicios educativos o a la promoción individual de la responsabilidad social, sino que debe existir un enfoque estratégico que aúne la satisfacción del estudiante al hacer coincidir mejor la calidad del servicio con las expectativas del mercado (Geryk, 2016). Dados estos resultados y los previos analizados (LópezAza, 2016; Vázquez et al., 2015) queda patente la necesidad de adaptar por rama académica la estrategia de marketing universitaria en el área de responsabilidad social para lograr instituciones de educación superior de más calidad y con estudiantes más satisfechos.

El alcance de los resultados obtenidos debe ser valorado teniendo presente ciertas limitaciones del estudio que abren caminos a futuras investigaciones en el área. En primer lugar, cabe apuntar la novedad del marco teórico utilizado en el planteamiento del modelo, particularmente en lo que atañe a las dimensiones de RSU. Así, son precisas nuevas investigaciones que repliquen los resultados obtenidos o profundicen en el refinamiento de los constructos propuestos y su medición en el contexto de la percepción estudiantil. En segundo lugar, sería recomendable comparar la muestra objeto de estudio con estudiantes de otras áreas a través de comparaciones intergrupales porque el procedimiento, por lo tanto, solo representa la percepción descrita por este grupo particular. En tercer lugar, sería interesante realizar estudios comparativos en otras universidades españolas, europeas y del resto del mundo, así como administrar el cuestionario a otros stakeholders como decanos, rectores y profesores para comparar los resultados y abrir el espectro de actuaciones. En cuarto lugar, la transversalidad del estudio limita las conclusiones del estudio a relaciones estadísticamente significativas por lo que un estudio longitudinales permitiría conocer la evolución de las percepciones de los estudiantes e incluso si estas se ven modificadas una vez acceden al mercado laboral ya que en la medición de la satisfacción la parte relacionada con los resultados solo es realmente perceptibles una vez que el estudiante finaliza su carrera y empieza a buscar empleo (Gruber, Fub, Voss y Gläser-Zikuda, 2010).Sería interesante a tales efectos completar el estudio con el análisis de las percepciones de los mismos estudiantes cuando hayan accedido al mundo laboral, viendo cómo se traduce su experiencia inicial en términos de práctica profesional responsable. En último lugar, sería adecuado introducir en el modelo otras variables como la fidelidad para conocer el alcance estratégico de esta propuesta.

\section{Bibliografía}

Abdullah, F. (2005). HEdPERF versus SERVPERF: The quest for ideal measuring instrument of service quality in higher education sector. Quality Assurance in Education, 13(4), 305-328

Alashloo, F. R., Castka, P. y Sharp, J. M. (2005). Towards understanding the impeders of strategy implementation in higher education (HE): A case of HE institutes in Iran. Quality Assurance in Education, 13(2), 132-147.

Andreassen, T. W. y Lindestad, B. (1998). Customer loyalty and complex services: The impact of corporate image on quality, customer satisfaction and loyalty for customers with varying degrees of service expertise. International Journal of Service Industry Management, 9(1), 7-23.

Arambewela, R. y Hall, J. (2008). A Model of student satisfaction: International postgraduate students from Asia. European Advances in Consumer Research, 8,129-135.

Balotsky, E. R. y Steingard, D. S. (2006). How teaching business ethics makes a difference: Findings from an ethical learning model. Journal of Business Ethics Education, 3,5-34.

Barclay, D., Higgins, C. y Thompson, R. (1995). The partial least squares (PLS) approach to causal modeling: Personal computer adoption and use as an illustration. Technology Studies, 2(2), 285-309

Ben, A. M. (2007). Identifying the needs of customers in higher education. Education, 127(3), 332

Bigné, E., Molinar, M.A. y Sánchez, J. (2003). Perceived quality and satisfaction inmultiservice organizations: The case of Spanish public services. Journal of ServicesMarketing 17(4), 420-442

Blázquez, J. J., Chamizo, J., Cano, E.I. y Gutiérrez, S. (2013). Calidad de vida universitaria: Identificación de los principales indicadores de satisfacción estudiantil.Revista de Educación, 362, 458-484

Boshoff, C. y Gray, B. (2004). The relationships between service quality, customer satisfaction and buying intention in the private hospital industry. Journal of Business Management $35(4), 27-37$

Brown, R. M. y Mazzarol, T. W. (2009). The importance of institutional image to student satisfaction and loyalty within higher education. Higher Education, 58(1), 81-95.

Burcea, M. y Marinescu, P. (2011). Students' perceptions on study: The Faculty of Administration and Business, University of Bucharest. Amfiteatru Economic, 1(29), 207-220

Caldwell, C. (2009). A ten-step model for academic integrity: A positive approach for business schools. Journal of Business Ethics, 92(1), 1-13.

Chin, W. W. (1998a). The partial least squares approach for structural equation modeling En A George (Ed) Modem Methods for Business Research (pp. 295-336). New Jersey: Laurence Erlbaum Associates.

Chin, W. W. (1998b). Commentary: Issues and opinion on structural equation modeling. MIS Quartely, 22(1), 7-16.

Christensen, L. J., Peirce, E., Hartman, L. P., Hoffman, W. M. y Carrier, J. (2007). Ethics, CSR, and sustainability education in the financial times top 50 global business schools Baseline data and future research directions. Journal of Business Ethics, 4,347-368

Comisión Europea. (2010). Comunicación de la Comisión Europa 2020: Una estrategia para un crecimiento inteligente, sostenible e integrador. [COM (2010) 2020 final - no publicada en el Diario Oficial, de 03-03-2010]. Bruselas: Autor.

Eklöf, J. A. (2000). European customer satisfaction index panEuropean telecommunication sector report - based on the pilo studies 1999. European organization for quality and European foundation for quality management. Stockholm, Sweden

Elkington, J. (1997). Cannibals with forks. Capstone.

Falk, R. F. y Miller, N. B. (1992). A primer for soft modeling. press. Akron, Ohio: The University of Akron.

Fernández, J. L., y Sanjuán, A. B. (2010). The Presence of Business Ethics and CSR in the Higher Education Curricula for Executives. Journal of Business Ethics Education, 7, 25-38. Fornell, C., Johnson, M. D., Anderson, E. W., Cha, J. y Bryant, B. E. (1996). The American customer satisfaction index: Nature, purpose, and findings. The Journal of Marketing, 60(4), 7-18.

Fornell, C. y Larcker, D. F. (1981). Evaluating structura equation models with unobservable variables and measurement error. Journal of Marketing Research, 18,39-50.

Garde-Sánchez, R., Rodríguez-Bolivar, M. P. y López-Hernández, A. M. (2013). Divulgación online de información de responsabilidad socialen las universidades españolas. Revista de Educación, Extraordinario, 177-209.

Geisser, S. (1974). A predictive approach to the random effect model. Biometrika, 61(1), 101-107. 
Geryk, M. (2016). Social Responsibility of the University. Gdansk Management College - Publishing House

Gournaris, S. P., Stathakopoulos, V. y Athanassopoulos, A. D. (2003). Antecedents to perceived service quality: An exploratory study in the banking industry. International Jounal of Bank Marketing, 21(4),168-190.

Gruber, T., Fub, S., Voss, R. y Gläser-Zikuda, M. (2010). Examining student satisfaction with higher education servces: Using a new measurement tool. Inernational Journal of Public Sector Management, 23(2), 105-123.

Hanaysha, J., Abdullah, H. H. y Warokka, A. (2011). Service quality and students' satisfaction at higher learning instituions: The competing dimensions of Malaysian universities competitiveness. Journal of Southeast Asian Research,1, 1-11.

Hartman, L. P. y Werhane, P. H.(2009). A modular approach to business ethics integration: At the intersection of the stand-alone and the integrated approaches. Journal of Busness Ethics, 90(3),295-300.

Hasan, H. F. A., Ilias, A., Rahman, R. A. y Razak, M. Z. A (2008). Service quality and student satisfaction: A case stuy at private higher education institutions. International B siness Research, 1(3), 163-175.

Huang, C,C., Yen, S. W., Liu, C. Y. yHuang, P. C. (2014) The relationship among corporate social responsibility, service quality, corporate image and purchase intention. national Journal of Organizational Innovation, 6(3), 68-84

Hulland, J. (1999). Use of partial least squares (PLS) in straegic management research: A review of four recent stu Strategic Management Journal, 20(2), 195-204.

Johnson, M. D., Gustafsson, A., Andreassen, T. W., Lervik, L. y Cha, J. (2001). The evolution and future of nationa customer satisfaction index models. Journal of Economic Psychology, 22(2), 217-245.

Joseph, M., Yakhou, M. y Stone, G. (2005). An educationa institution's quest for service quality: Customers' perspective. Quality Assurance in Education, 13(1), 66-82.

Kilbourne, W.E., Duffy, J.A., Duffy, M. y Giarchi, G. (2004) The applicability of SERVQUALin cross-national measurements of health-care quality. Journal of Services Marketing, 18(7), 524-33.

andrum, H., Prybutok, V. R. y Zhang, X. (2007). A compatison of Magal's service quality instrument with SERVPERE Information \& Management, 44(1), 104-113.
Larrán, M. y Andrades, F J. (2014). Determinants of corporate social responsibility and business ethics education in Spanish universities. Business Ethics: A European Review, 23(2), 139-153.

Loureiro, S. y González, F. J. (2012). DUAQUAL: Calidad percibida por docentes y alumnos en la gestión universitaria/ DUAQUAL: The quality perceived by teachers and students in university management. Cuadernos De Gestión, 12(1), 107-122

López-Aza, C. (2016). Responsabilidad y sostenibilidad: situación actual y perspectivas de tratamiento en la educación supenior univertiaria. Tesis doctoral, Universidad de León.

Maignan I., Ferrell O.C. (2001). Corporate citizenship as a marketing instrument: Concepts, evidence and research directions. European Journal of Marketing, 35, 3-4.

Mandhachitara R. y Poolthong Y. (2011). A model of customer loyalty and corporate social responsibility. Journal of Services Marketing, 25(2), 122-133.

McDonald, G. M. (2004). A case example: Integrating ethics into the academic business curriculum. Journal of Business Ethics, 54(4), 371-384.

Mele, R. (2003). Economia e gestione delle imprese di pubbliciservizitra regolamentazione e mercato. Cedam, Padova.

Moon, J. y Orlitzky, M. (2011). Corporate social responsibilty and sustainability education: A trans-atlantic comparison Journal of Management and Organization, 17(5), 583-603.

Munteanu, C., Ceobanu, C., Bobâlca, C. y Anton, O. (2010). An analysis of customer satisfaction in a higher education context. InternationalJournal of Public Sector Management, 23(2), 124-140,

O'Neill, M. A. y Palmer, A. (2004). Importance-performance analysis: A useful tool for directing continuous quality mprovement in higher education.Quality assurance in education, 12(1), 39-52.

Perić, J. y Delić, A. (2016). Developing social responsibility in Croatian Universities: a benchmarking approach and an overview of current situation. International Review on Public and Nonprofit Marketing, 13(1), 69-80,

Plungpongpan, J., Tiangsoongnern, L., y Speece, M. (2016). University Social Responsibility and Brand Image of Private Universities in Bangkok International Journal of Educationa Management,30(4), 571-591
Prasad, R. K. y Jha, M. K. (2013). Quality measures in higher education: A review and conceptual model. Journal of Research in Business and Management, 1(3), 23-40.

Qureshi, T. M., Shaukat, M. Z. yHijazi, S. T. (2010). Service quality SERVQUAL model in higher education institutions. What factors are to be considered? Interdisciplinary Journal of Contemporary Research in Business, 2(5), 281-290.

Ringle, C. M. Wende S. y Will, A. (2005) SmartPL S-Version 2.0.Hamburgo: Universität Hamburg.

Russell, M. (2005). Marketing education: A review of service quality perceptions among international students. International Journal of Contemporary Hospitality Management 17(1), 65-77.

Sánchez-Hernández, M.I. y Mainardes E. M. (2016). University social responsibility: a student base analysis in Brazil. International Review on Public and Nonprofit Marketing 13, 151-169

Setó-Pamies, D., Domingo-Vernis, M., y Rabassa-Figueras, N. (2011). Corporate Social Responsibility in Managemen Education: Current Status in Spanish Universities. Journal of Management and Organization, 17 (5), 604-620.

Shekarchizadeh, A., Rasli, A. y Hon-Tat, H. (2011). SERVQUAL in Malaysia universities: Perspectives of international students. Business Process Management Journal, 17(1) 67-8

Sobczak, A., Debucquet, G., y Havard, C. (2006). The Impact of Higher Education on Students' and Young Managers' Perception of Companies and CSR: An Exploratory Analysis. Corporate Governance, 6(4), 463-474.
Stone, M. (1974). Cross-validatory choice and assessmen of statistical predictions. Journal of the Royal Statistical Society. Series B (Methodological), 111-147.

Tan, K. C. y Kek, S. W. (2004). Service quality in higher education using an enhanced SERVQUAL approach. Quality in Higher Education, 10(1), 17-24.

Tennenhaus, M., Vinzi, V., Chatelin, Y-M. y Lauro, C (2005). PLS path modeling Computational Statistics and Data Analysis, 48(1), 159-205.

Vallaeys, F. (2006). Brief Theoretical Framework of University Social Responsibility. Universidad Católica de Perú. Recuperado de: http://blog.pucp.edu.pe/item/4880/bre-marco-teórico-de-rsu.

Vallaeys, F. (2014). La responsabilidad social universitaria Un nuevo modelo universitario contra la mercantilización. RIES Revista Iberoamericana De Educación Superior, 12(5), 105-117

Vázquez J.L.; López-Aza C.: Lanero, A. (2015). University Social Responsibility as Antecedent of Students' University Experience, Responsibility and Sustainability, 3(3), pp $33-46$

Wetzels, M., Odcerkerken-Schröder, G.y Van Oppen, C (2009). Using PLS path modelling for assessing hierarchical construct models: Guidelines and empirical illustration. MIS Quarterly, 33(1), 177-195.

Zeithaml, V. A. y Bitter, M.J. (2002).Il Marketing dei Servizi. Milano: McGraw Hill. 